\title{
Hydroforming of locally heat treated tubes.
}

\author{
Lorenzo lorio ${ }^{1}$, Ernesto Maspero ${ }^{2}$, Matteo Strano ${ }^{3}$ \\ ${ }^{1}$ MUSP Lab, Piacenza, Italy, lorenzo.iorio@musp.it \\ ${ }^{2}$ Fonderia Maspero srl, Monza, Italy, ernesto.maspero@maspero.it \\ ${ }^{3}$ Politecnico di Milano, Dipartimento di Meccanica, Milan, Italy, matteo.strano@polimi.it
}

\begin{abstract}
In tube hydroforming, the process chain can be very long as it may involve several pre-forming operations (e.g. bending, crushing, end forming, etc.) which are usually followed by an intermediate annealing stage. Conventional annealing is performed in batches and it is often perceived as a long, relatively expensive and non-environmentally friendly operation. For this reason, in this paper local intermediate heat treatment is proposed as a promising alternative solution, in order to reduce the throughput process time. The study has been carried out on a real tubular motorcycle part, by performing both experiments and numerical simulations, in order to verify whether local annealing can be an effective substitute of conventional global annealing. Several alternative ways of locally heat treating an Al6060 tube right before hydroforming have been investigated. The results demonstrate that a feasible solution can be found, with local heat treatment of relatively small portions of the tube.
\end{abstract}

Keywords: tube hydroforming, intermediate annealing, local heat treatment, Al6060, FEM

\section{Introduction}

Local heating of blanks by either induction, resistance, infrared or laser beam has been frequently proposed as an option for innovation especially in tube and sheet metal forming processes, with some applications in bulk metal forming operations, too [1], [2].

Local heating concepts have been often developed as an in-process conditioning technique, i.e. applied during the forming operation. The most frequent purpose of in-process local heating approaches is to enhance formability of materials, i.e. to reduce the risk of fracture for difficult-to-form alloys (e.g. magnesium alloys [3], aluminium alloys [4], etc.).

Additionally, local heating can be aimed at reducing the strength and increasing ductility of areas interested by severe deformations, so that forming forces are reduced and the final shape of formed parts better calibrates the die shape [5], [6]. Accuracy of final shape is also a primary concern of heat assisted incremental forming processes, where dieless tooling setups are often used and heating can be a way for reducing undesired deflections and springback [7]. The reduction of springback by in-process local heating is useful also in other sheet forming processes, such as deep drawing [8] and bending [9], [10].

In bending processes, a concentrated thermal power sometimes represents not only a way for process improvement, but the actual driving mechanism that allows plastic deformation. In tube and pipe induction bending [11], local heating by an induction ring which is placed immediately before the bending region allows for the tube to bent only where required, with a very simplified, nearly dieless tooling setup. Laser bending of sheets is another technique where a nearly dieless forming operation is made possible by a concentrated thermal power [12].

In-process local heating is often employed in free [13] or dieless forming operations, such as dieless incremental forming [14] or dieless tube bending [15]. The reason is that when there is large contact between the blank and the tools, conduction heat transfer occurs [16] and, therefore, the temperature of all tools must be carefully and locally controlled, otherwise the local effects disappear. As a consequence, in order to maintain the desired thermal gradients in the workpiece, complex differential heating/cooling strategies of punches, moulds and other tools are often required [17]. Even when the heat conduction problems due to a complete contact to a die or mould are negligible, the forming workpiece must be very accessible from at least one of its sides, such as in conical spinning [18] or in free bulge hydroforming [19]. This is because the most rapid and effective heating techniques (laser beam and induction) require close but contactless interaction with the interested region of the workpiece. As a matter of fact, when processes are involved, that require a continuous contact of the part with a completely closed die, such as tube hydroforming, in-process local or differential heating of the part becomes virtually impossible. A viable alternative in these cases is to 
use local heating as a preliminary heat treatment, implemented before the process starts, in order to selectively change the ductility of the workpiece.

Local heating as a heat treatment has been suggested by some authors: Järvenpää and co-authors studied how a local laser heat treatment can be used to produce ultrafine-grained austenite structures in an austenitic steel [20]; Capello and Previtali studied the effect of different typologies of surface treatment by diode laser on the local formability of dual phase steel [21]; Neugebauer and Ahnert showed how differential hardness tracks and patterns of relatively small width $(0.5$ to $5 \mathrm{~mm})$ can be induced on steel sheets by laser heating [22]. The above mentioned studies concentrate on the material properties and on the consequences of different heating strategies. One step forward has been moved by Sulzberger and co-authors, towards application of this concept in a real forming process; they proposed the design of a benchmark part intended for reverse deep drawing operations, and performed a simulation study [23]. Staud and Merklein proposed the original concept of Taylor Heat Treated Tubes (THTB), testing the concept on aluminium tubes of the 6000 series [24], as in the present paper.

In this paper we demonstrate how local annealing of a tube can be an interesting option in tube hydroforming: a) in order to reduce the maximum pressure required for final geometrical calibration; $b$ ) in order to reduce the risk of tube fracture during the process, avoiding complete intermediate annealing operations, which are expensive both from an economical and environmental point of view. In hydroforming, it is well known that the calibration pressure and the corresponding press closure force both depend on the material properties, the wall thickness of the tube and the value of the minimum concave internal die radius [25]. Therefore, reducing the strength of the material only in the regions which will be involved in calibration of small radii is an option for reducing the hydraulic pressure and press tonnage requirements. Before being hydroformed, most tubular parts undergo preliminary forming operations (bending, nosing, crushing) which strain harden the material and reduce its formability. For this reason, an intermediate annealing operation is frequently performed before the part enters the hydroforming press [26]. A conventional full annealing operation is time consuming, it is often performed outside the shop where hydroforming takes place, it is relatively costly and it is not friendly to the environment. On the contrary rapid and local heating, e.g. by means of a laser beam, could allow to reduce the total energy spent in the process and the total processing time and could be more easily integrated into an automated process.

The advantages of local intermediate heat treatment in a hydroforming process chain of tubular motorbike part, made by aluminium alloy, will be shown in this paper. In Section 2, the current standard processing cycle of the reference part, which includes three main stages (preforming, annealing, hydroforming) will be shown. In Section 3, an FEM model is presented and its fidelity is demonstrated by comparing the numerical results to the experimental results of cycles performed with and without the annealing stage. Once the FEM model has been validated, in Section 4 it is used in order to demonstrate how the results of local heat treatments compare to the results of a full intermediate annealing stage.

\section{Description of process design, equipment and test results}

The reference part, which is a tubular motorcycle component, is shown in Figure 1. It is made by aluminium alloy Al 6060, outer diameter OD $80 \mathrm{~mm}$, initial wall thickness $t_{0} 2.2 \mathrm{~mm}$, yield stress $63 \mathrm{~N} / \mathrm{mm}^{2}$, Tensile strength $96 \mathrm{~N} / \mathrm{mm}^{2}$ and maximum elongation $28.4 \%$. As the figure also shows, the as-received extruded tube, in an annealed condition, is first made conical at both ends by means of a swaging process [27]. As the tube ends undergo severe thickening, surface damage (Figure $2 b$ ) and hardening, the tube is subsequently fully annealed by furnace and then cooled at room air temperature.

Then, the tube is placed in a hydroforming die, its ends are sealed by axial punches, and it is hydroformed with a maximum internal pressure of about $62 \div 65 \mathrm{MPa}$ with a hydroforming time of about $15 \mathrm{~s}$. If the internal pressure exceeds $65 \mathrm{MPa}$, the dies would open, given the tonnage of the single effect hydraulic press 11000 $\mathrm{kN}$. used for the process. Hydroforming dies are built with an alloyed tool steel 56NiCrMoV7. As far as the lubrication is concerned, the dies are polished and the tubes are abundantly lubricated with liquid lubricant (mineral oil).

The process has been tried out with a testing phase of about 100 tubes, in order to: 1 ) select the loading curves (pressure and feed), 2) define the need for intermediate annealing steps. A feasible and robust process has been designed with an experience-driven trial and error procedure. The selected process and the discarded design options are described as follows. 
LOADING CURVES. Hydroforming is performed under self-feeding [25] conditions for the axial cylinders. This is a typical case of aluminium hydroforming, when axial feed must be kept to a minimum, in order to prevent wrinkling and further damage of tube ends. In other words, the machine does not control the position of the two axial cylinders, which are clamped from the inside to the tube ends in order to prevent leaking but are idle on the axial movement. There are several reasons for a very limited axial feed is applied: a) the tube ends have a short straight guiding zone; b) the tube ends have already been significantly thickened and damaged by the swaging operation; c) the purpose of hydroforming in this case is more of shaping and calibrating, with limited perimeter expansion about of maximum $6 \%$.

The hydroforming loading curves can be divided in three phases, described as follows:

1. Filling: the water fills the tube for 2 seconds until a pressure of $6 \mathrm{MPa}$ is reached, with a tube shrinking of about $0.03 \mathrm{~mm}$ in axial direction (and corresponding displacement of axial punches).

2. Forming: it is the true hydroforming phase, when the tube takes the shape of the dies; this stage needs about 15 seconds and the pressure linearly increases from 6 bar to $62 \mathrm{MPa}$ bar and the tube shrinks of about $1.04 \mathrm{~mm}$ axially. As stated above, the axial stroke of the cylinders is negligible.

3. Calibrating: in this phase there is a pressure increasing needed for filling all die cavities and calibrating the tube for 3 seconds, until $65 \mathrm{MPa}$ pressure is reached; virtually no axial tube shrinking occurs.

After hydroforming the damaged ends are trimmed away and the tube is prepared for subsequent assembling operations.

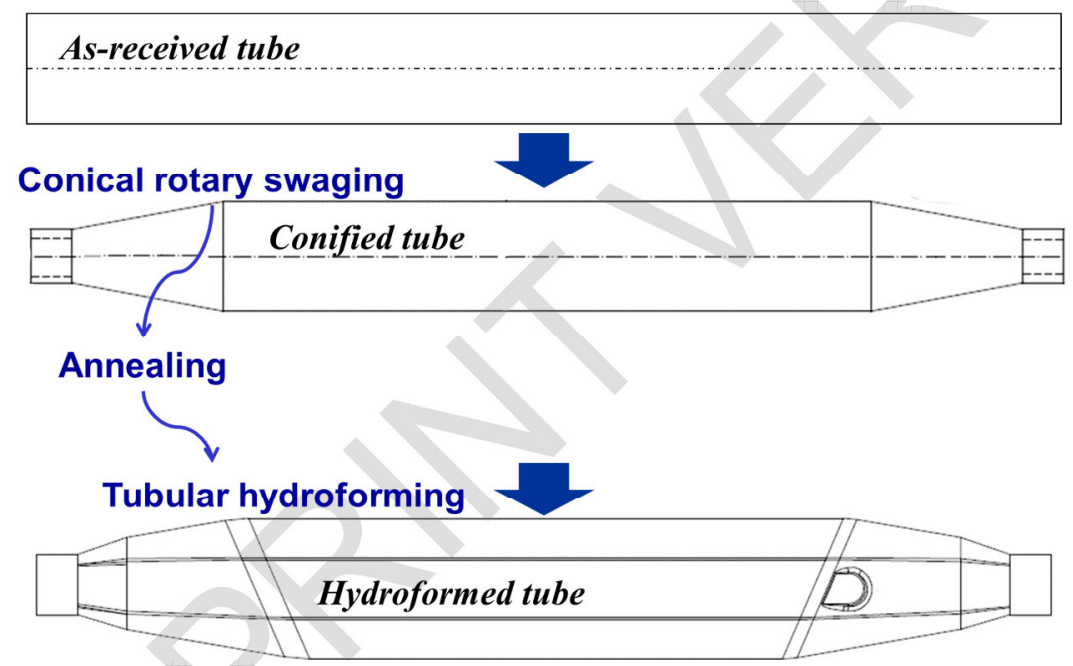

Figure 1: reference hydroformed part and process chain

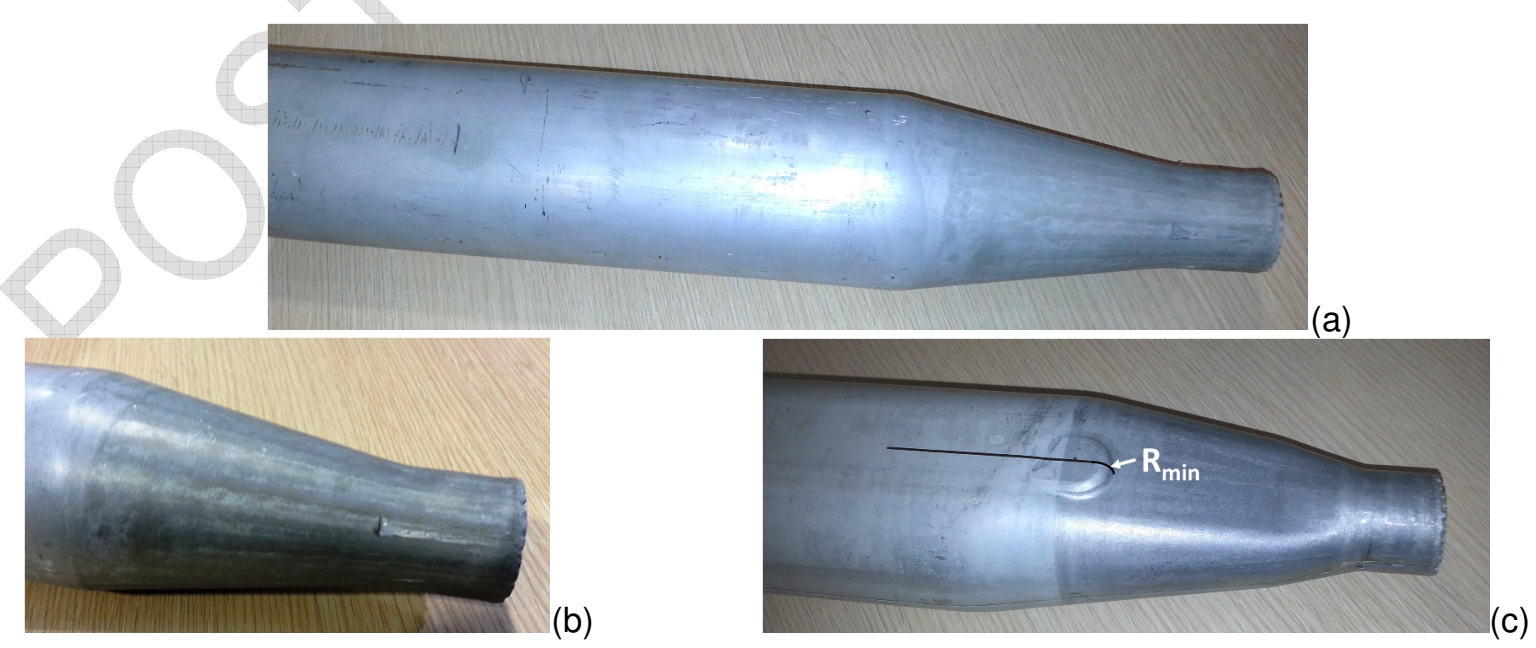

Figure 2: images of a portion of the tube after swaging (a); detail of conical region after swaging (b); hydroformed tube (c) 
ANNEALING OPTIONS. In case an intermediate annealing stage is not performed, the results of the hydroforming stage are unsatisfactory as the part often fails by fracture. The occurrence of fracture for nonannealed tubes is close to $90 \%$, mostly at the location shown in Figure 3a. Furthermore, even if the part does not fail (about $10 \%$ of the tested samples), the geometrical feature on top of the flat surface of the final tube, a sort of semi-dome, is insufficiently calibrated at the prescribed maximum pressure, as evident from the comparison of Figure $3 \mathrm{~b}$ with Figure 2c.

As explained above, the route of increasing axial feed is not feasible for improving formability of this part. The only available option is to anneal the tube. In fact, if a full conventional annealing treatment is performed in a furnace (at over $350^{\circ} \mathrm{C}$ for nearly 3 hours), the process implemented with the loading curves described above is $100 \%$ safe and the dome is correctly calibrated.

In order to avoid conventional annealing, a possible innovative technique would be to perform a localised heat treatment of the tube in the most critical regions.

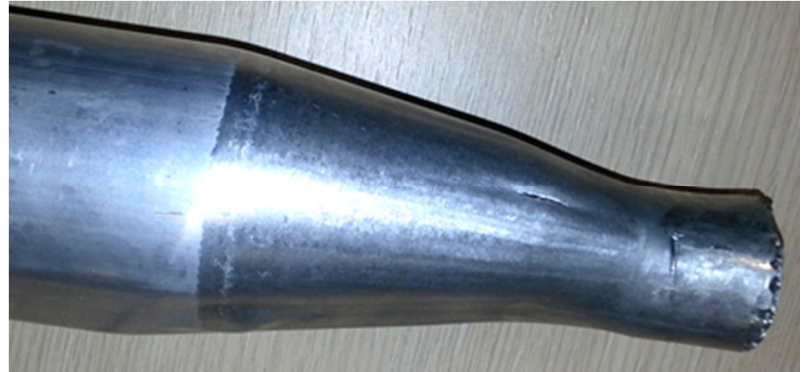

(a)

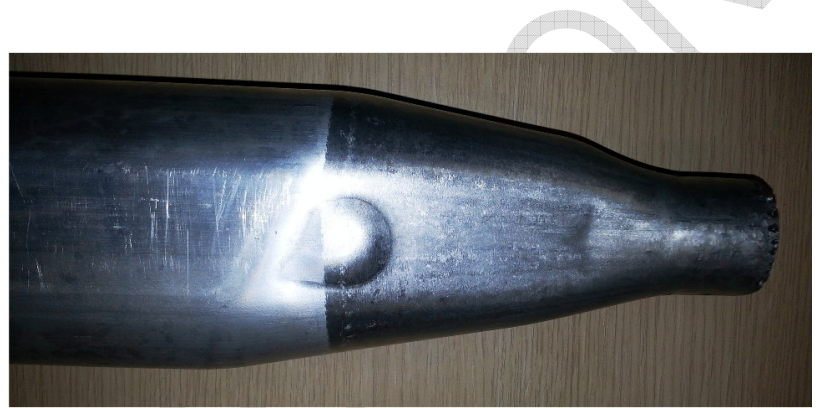

(b)

Figure 3: location of fracture on the tube when annealing is not performed (a); insufficient calibration of the semi-dome (b)

\section{FEM modelling of the process}

\subsection{Description of the FEM model}

The FEM model has been implemented with the commercial code PAM-STAMP 2G V2012, which allows to define the geometry of the tube directly in the graphical unit interface, in a parametric way. In the software, the tube module allows to define two kinds of objects:

- tools: the mesh is automatically defined by the program with triangular and quadratic shell elements in order to better approximate the rigid surface of the tool.

- Tube: defined with diameter, length and dimension of quad shell elements.

The diameter of tube, geometrically defined by its middle surface, is equal to $77.8 \mathrm{~mm}$, the thickness is 2.2 $\mathrm{mm}$ and the total I nitial length is $670 \mathrm{~mm}$. The surface area of each initial element is $4 \mathrm{~mm}^{2}$ and the total number is 41205 ; this quantity is also refined during the simulation with automatically refining process with two refinement steps allowed for each forming stage. Every refinement step split one quadrangular element into four smaller quadrangular shells. The constitutive law used for material definition is the Krupkowsky's formulation:

$\sigma=K \cdot\left(\varepsilon_{0}+\varepsilon_{p}\right)^{n}$

whose coefficients, obtained from tensile test data, are listed in Table 1.

\begin{tabular}{l|r}
$\boldsymbol{K}$ & $0.2577[\mathrm{GPa}]$ \\
\hline $\boldsymbol{n}$ & 0.251 \\
\hline $\boldsymbol{\varepsilon}_{0}$ & 0.0006
\end{tabular}

Table 1: Krupkowsky law coefficients for al6060. 
The setup starts with the cylindrical tube that is gradually reduced with three subsequent reducing stages. These operations are an approximation of the real swaging process, in order to simplify the kinematics of the model and reduce the simulation time. In each stage, a conical die, modelled as rigid body, translates toward the tube in order to operate a gradual diameter reduction of the tube ends. The reducing stages shape the tube from zero to a 20 degrees conicity, with intermediate values at 10 and 16 degrees.

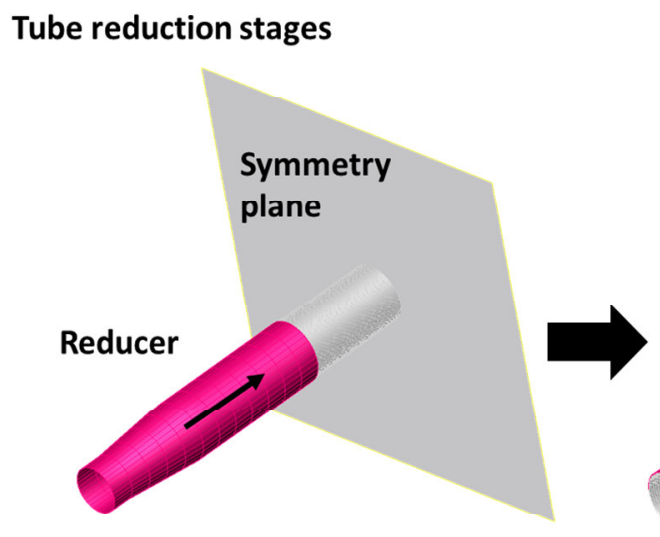

Start point

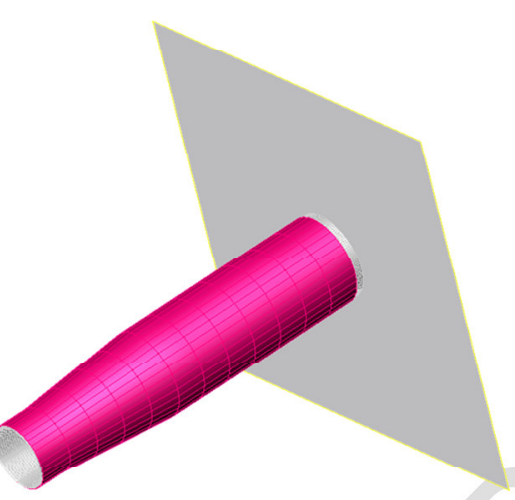

End point

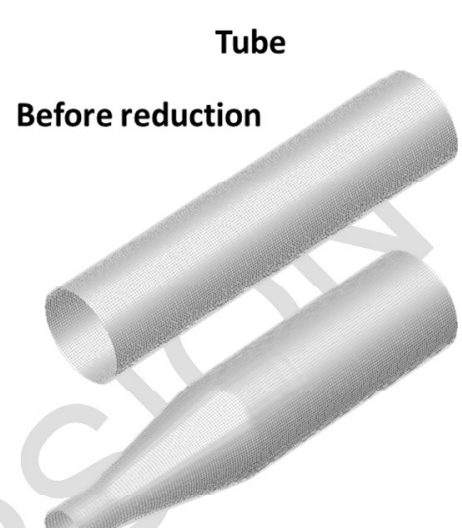

After reduction

Figure 4: scheme of reducing stages, where the reducer translates axially

After the 3 reducing stages, a closing stage is defined in order to simulate the tube crushing before the hydroforming process. In the closing stage the hydroforming top die, a rigid body, translates downwards with constant velocity in order to close against the bottom die.

At the end of the closing process, the hydroforming stage is performed, with the internal pressure applied on the shell elements of the tube. The loading conditions described in Section 2 have been used for all simulations.
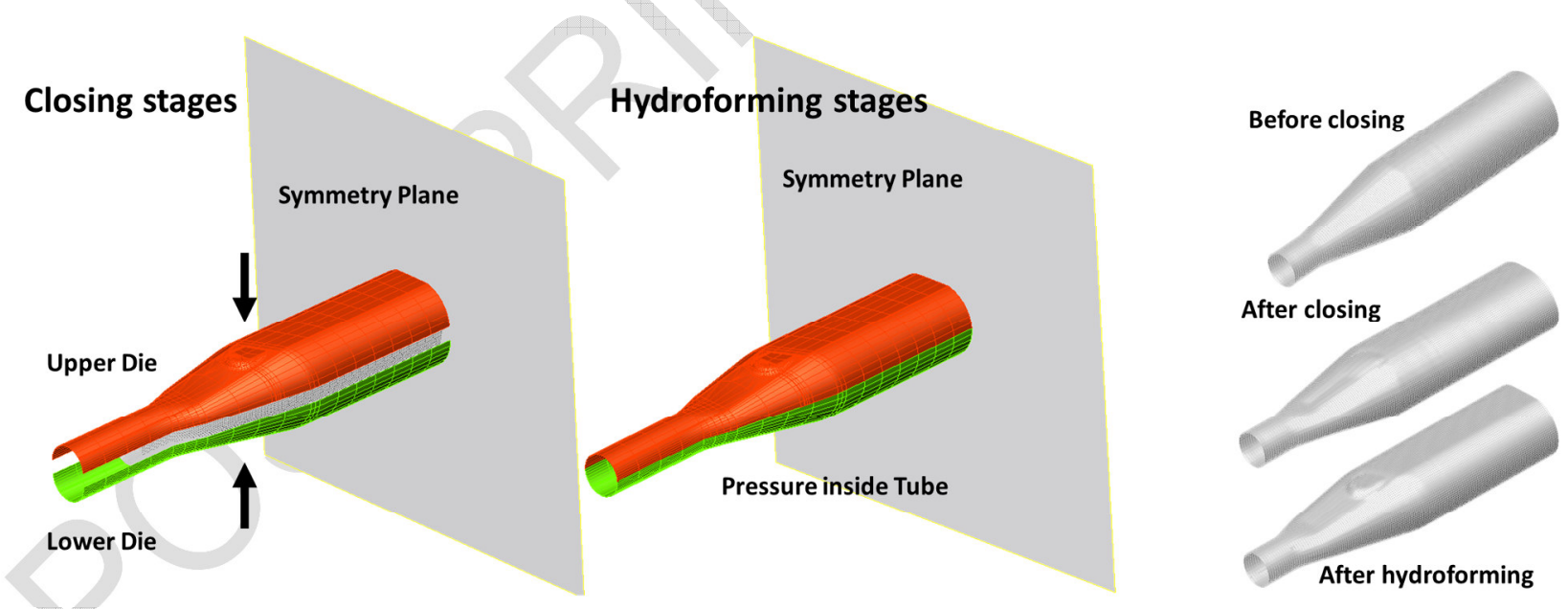

Figure 5: Scheme of closing and hydroforming stages.

The contact algorithm between the objects is automatically defined by the software taking in account a Coulomb friction coefficient of 0.05 , in order to simulate an abundant and uniform oil lubrication condition.

The procedure described above concerns the FEM model of the process with no intermediate annealing. In order to simulate the process with global annealing, a so-called object "picking" operation is required before the closing stage. Annealing is performed numerically by multiplying the stresses and strains by zero and keeping the geometry and thickness distribution of the tube as the end of the reducing stage.

The local annealing process requires two picking operations on different portions of the tube: a smaller picked portion undergoes annealing; the rest of the tube proceeds to the closing stage without annealing. 
Clearly, the two subsets are merged by their nodes before closing and hydroforming stages may start. Each stage is calculated with 8 parallel processors, the whole simulation chain requires about 37 hours to run.

\subsection{Validation of the FEM model}

As explained above, the simulation of the swaging process has been greatly simplified with respect to the real process, in order to speed up the total simulation time. Nevertheless, a comparison of the thickness profile of the simulated and experimental parts (Figure 6), measured along an axial cross section of the conical portion of the tube, shows that the actual thickness is well predicted in the conical region (points 5 to 15 in Figure 6) and slightly overestimated in the cylindrical region (points 1 to 5). However, the cylindrical region is not further expanded by the subsequent hydroforming stage, and it only serves as a guiding zone for the axial punches. The conical part of the tube has been cut in four parts as shown in Figure 6 . The thickness has been measured in 15 points over each side of the trimmed tube and all obtained measurements have been averaged, in order to obtain the plotted profile.

As far as the hydroforming stage is concerned, two phenomena must be reliably simulated and predicted: the risk of fracture of the tube (when no intermediate annealing is performed) and the geometrical profile of the semi-dome shown in Figure 2c.

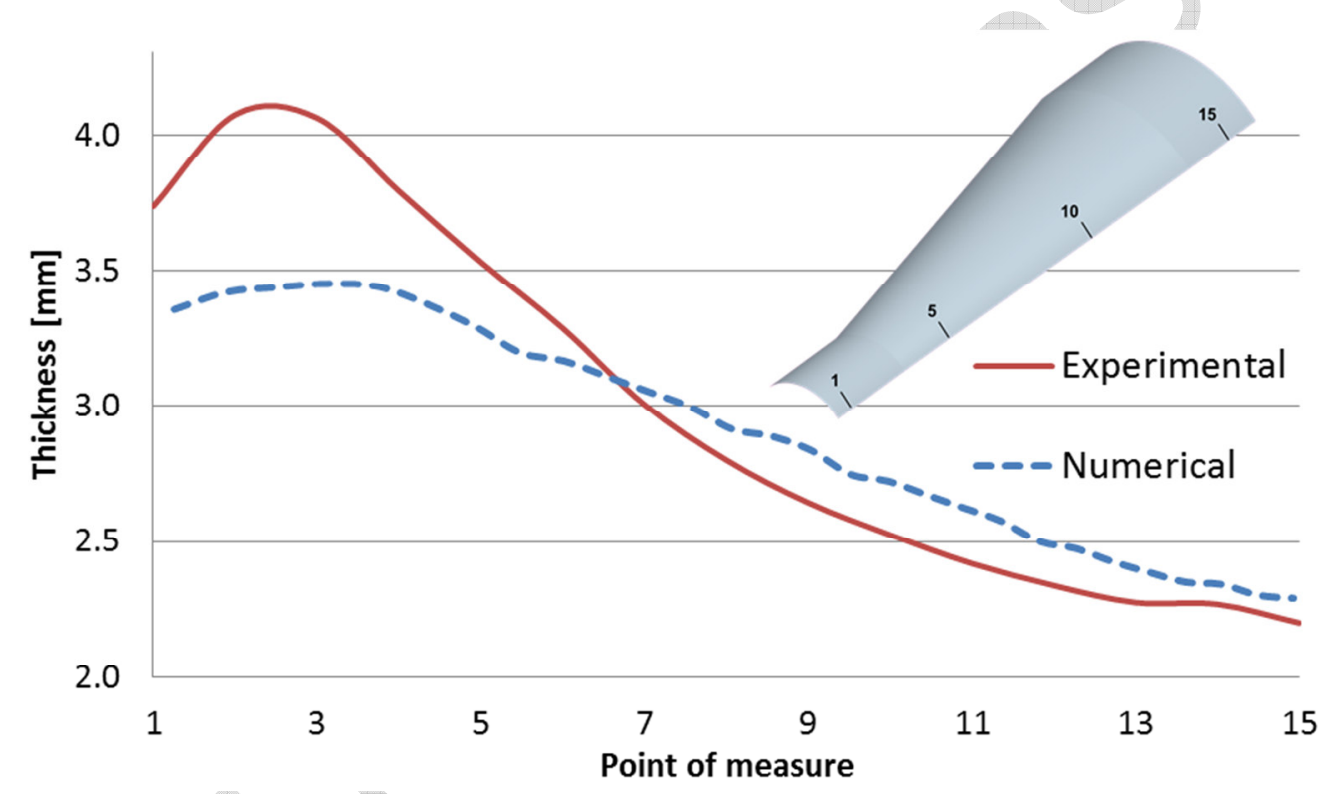

Figure 6: comparison of average experimental and simulated thickness profile after swaging operation; a quarter of a tube is shown on the figure, with indication of the location of measurement points.

An interesting comparison between the simulation and the real part can be evaluated on the hydroformed tube, in case no intermediate annealing is performed. In Figure 7 the plot of principal planar membrane strains on the final part is shown. An FLC (Forming Limit Curve) is plotted too, calculated according to the Keeler's empirical model. Each element of the tube mesh is colored differently according to the distance from this line, and points which are very close to the limit or beyond this limit are colored in red or orange. This colors in other words roughly indicate the risk of fracture of the tube and, quite evidently, the larger risk of fracture is recorded by the FEM exactly at the same location where the real part is fractured. Furthermore, both in experiments and simulations the bursting pressure amounts to approximately $55 \mathrm{MPa}$.

Fracture is located the beginning of the conical region, very close to the die parting line. However, there is no pinching effect both on the real and the simulated part. The proximity of fracture to the parting line part is therefore only a coincidence. The occurrence of fracture on the conical zone is rather caused by the severe expansion and deformation of the cross section where bursting occurs. In Figure 8 the tube is cut in three critical cross sections: the cross section where fracture occurs (called "fracture section" in the figure), the cross section where the conical region ends ("neck section") and a cross section at the middle axial position ("uniform section). These three cutting sections have been studied in terms of expansion ratio between the 
perimeters after and before hydroforming. In Table 2, the resulting expansion ratios are presented. The "uniform section", as shown in Table 2, presents the highest deformation ratio with a perimeter increase of about $6 \%$. The "fracture section" has a similar expansion (5\%) but the radius of curvature is clearly narrower at the parting line and this generates larger and quicker straining.

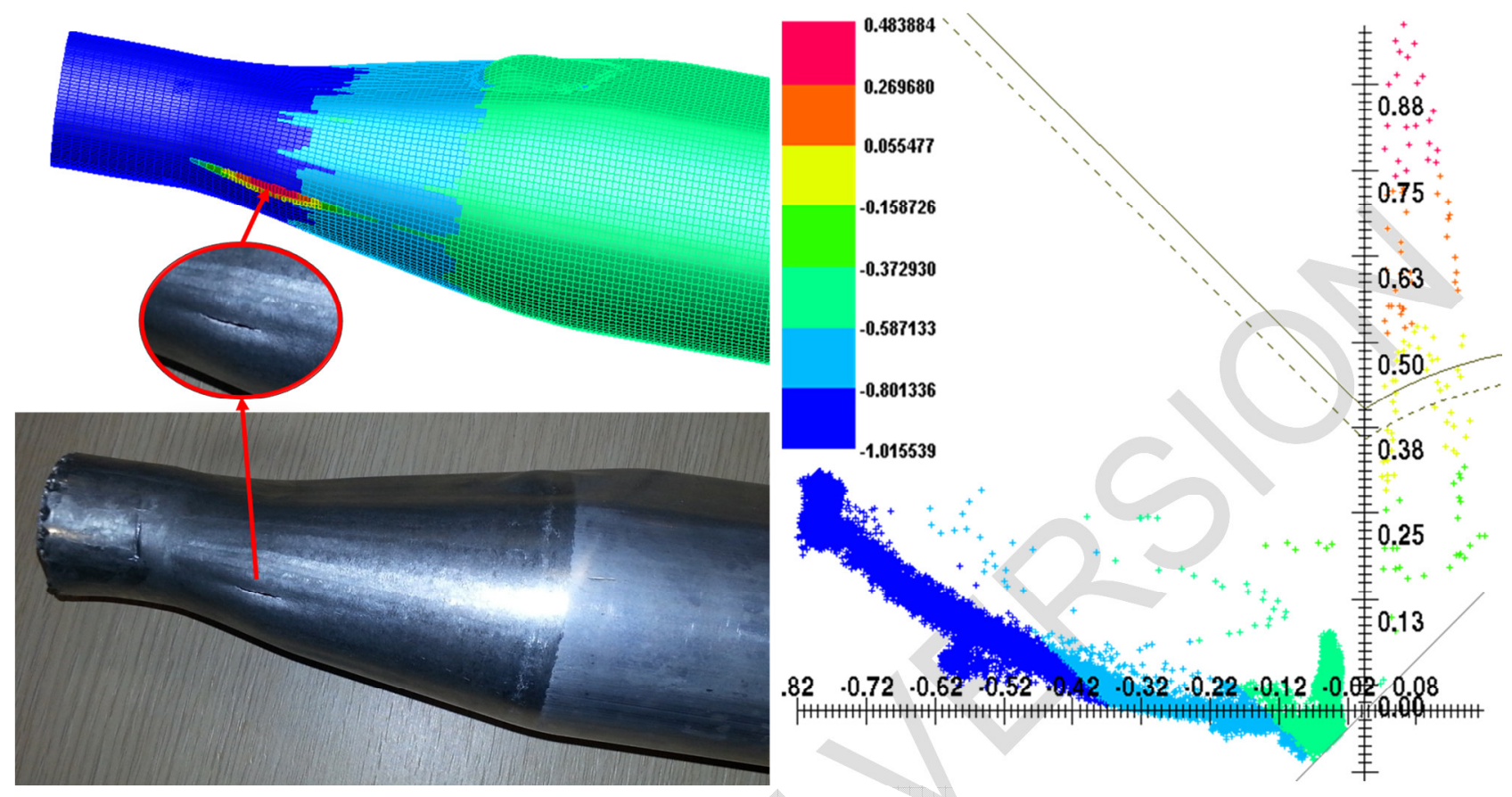

Figure 7: location of fracture on the tube when annealing is not performed well predicted by the simulation through an empirical "Keeler" limit curve and corresponding forming limit diagram.

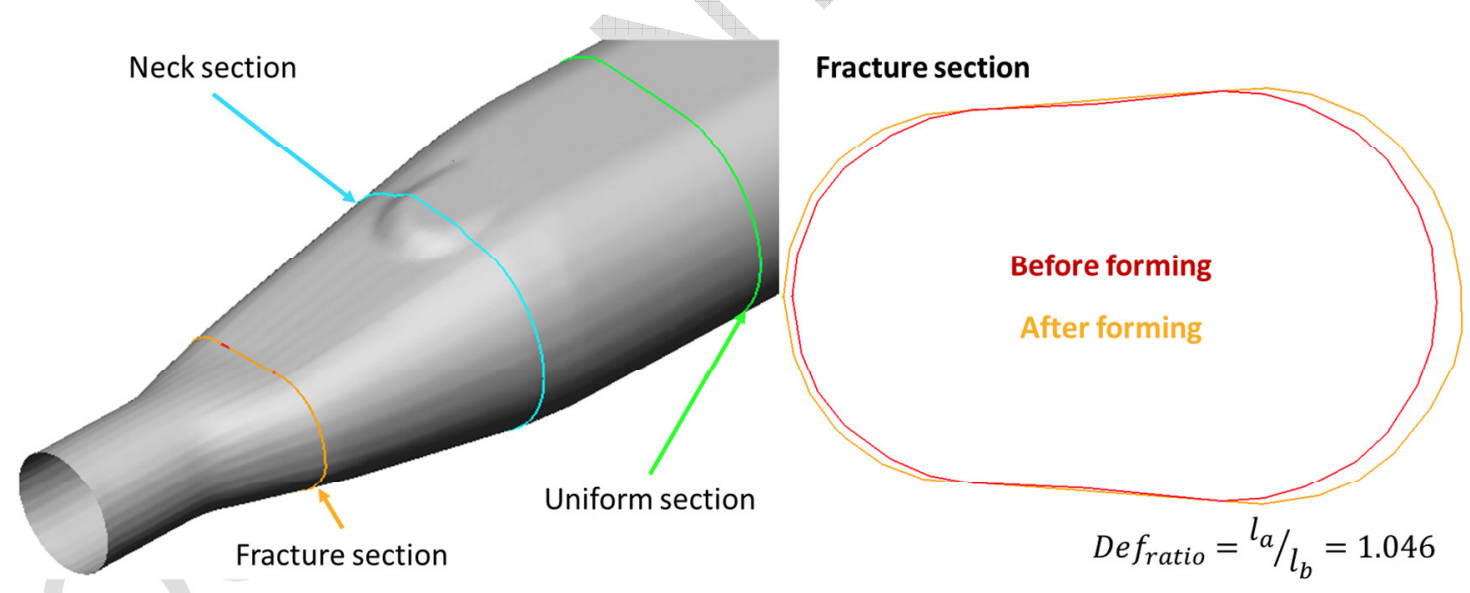

Figure 8: tube sections studied (left) and critical section (right).

\begin{tabular}{|c|c|c|c|c|c|}
\hline \multicolumn{2}{|c|}{ Fracture section } & \multicolumn{2}{c|}{ Neck section } & \multicolumn{2}{c|}{ Uniform section } \\
\hline Before & After & Before & After & Before & After \\
\hline $147.428 \mathrm{~mm}$ & $154.220 \mathrm{~mm}$ & $229.723 \mathrm{~mm}$ & $232.348 \mathrm{~mm}$ & $243.948 \mathrm{~mm}$ & $258.054 \mathrm{~mm}$ \\
\hline Def $_{\text {ratio }}$ & $\mathbf{1 . 0 5}$ & Def $_{\text {ratio }}$ & $\mathbf{1 . 0 2}$ & Def $_{\text {ratio }}$ & $\mathbf{1 . 0 6}$ \\
\hline
\end{tabular}

Table 2: sections length [mm] and expansion ratios at three relevant cross sections.

The profile of the tube after calibration has been measured along the cross section indicated by the black line in Figure $2 \mathrm{c}$ and especially the obtained minimum radius of curvature $\mathrm{R}_{\min }$ has been carefully measured, 
on the outer surface of the tube. $R_{\min }$ is the variable of interest in order to estimate the occurrence of insufficient calibration: when pressure is not enough with respect to the material strength and the final thickness, the value of $R_{\min }$ will be larger than expected. In Table 3, a comparison of the simulated and experimental values is given. The experimental radii have been evaluated on two components at the four ends and then the obtained values have been averaged. The simulation overestimates $R_{\min }$ of a nonnegligible (approximately $+35 \%$ ) error in both simulations, with and without intermediate annealing. However, this error is systematic and it allows the comparison of the effect of different strategies on shape calibration.

\begin{tabular}{|l|c|c|}
\hline & $F E M$ calculated $R_{\min }[\mathrm{mm}]$ & Experimental $R_{\min }[\mathrm{mm}]$ \\
\hline With intermediate annealing & 7.8 & 5.6 \\
\hline Without annealing & 16.0 & 12.2 \\
\hline
\end{tabular}

Table 3: comparison of experimental and simulated minimum radius on the tubes

\section{Comparison of global and local annealing strategies}

\subsection{Risk of fracture}

In this Section the potential outcome of local annealing techniques is investigated, by means of FEM simulations. Four options for local annealing have been tested, trying to heat treat the tube only on small critical regions or patches where defects eventually may develop. These options are described in Figure 9, labeled with letters from b) to e), listed with increasing surface area of the treated regions. In case b) only a small ring around the bursting region is treated and a crown where the calibrated dome will eventually form; in case c) a large approximately square patch is treated around the dome to be formed and a rectangular strip where fracture may develop; in case d) the tube is treated similarly to case c) but with a narrower patch around the dome and a thicker strip around the potential fracture line; finally, in case e) the entire conical portion of the tube is heated.

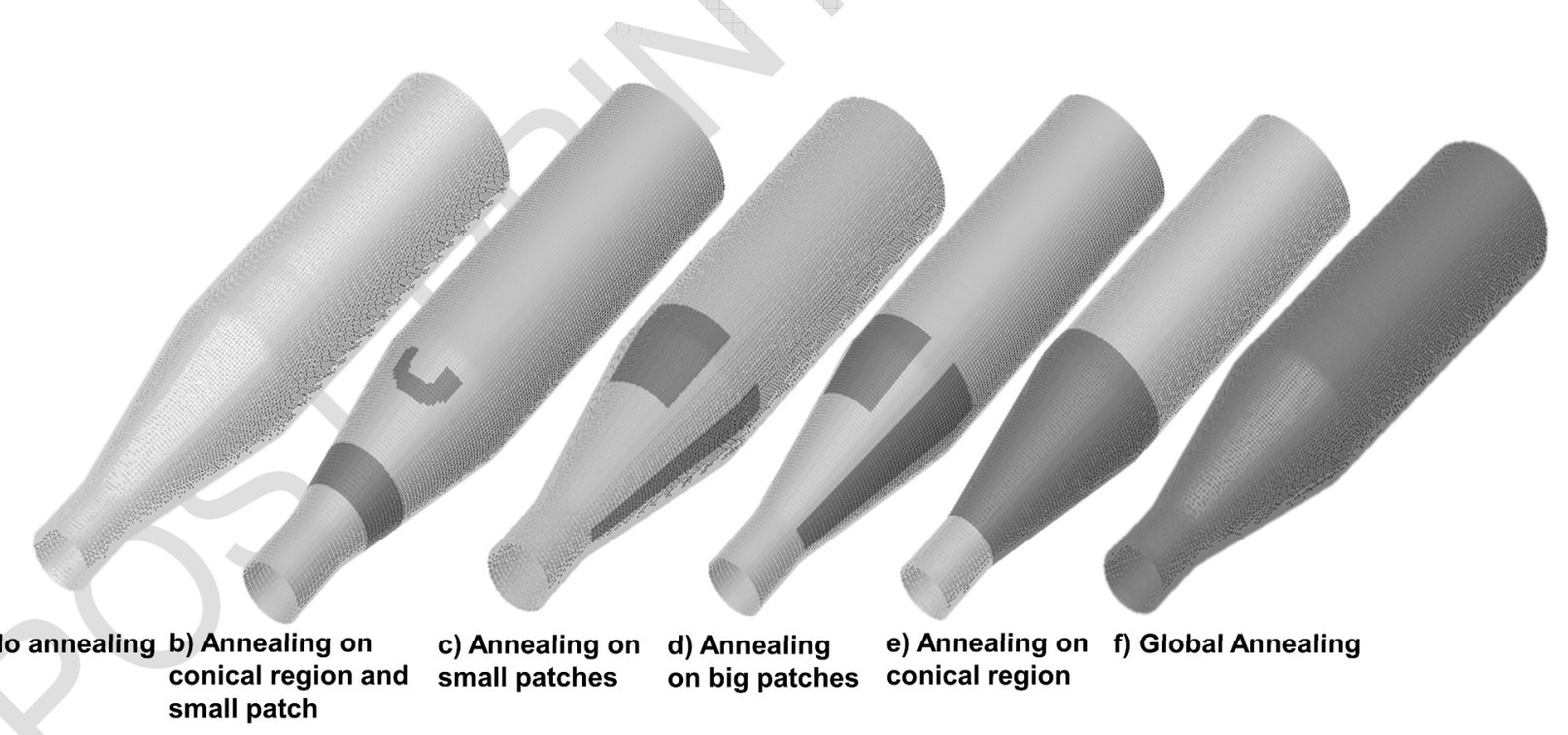

Figure 9: different annealing strategies used in simulations.

Figure 9 also shows two additional extreme cases, labeled respectively as cases a) and f). They have been already described in the previous Section: hydroforming with and without global intermediate annealing of the workpiece after the swaging operation. Considering the risk of fracture at the die parting line, case a), as already demonstrated in Fig. 5 and as confirmed by the experiments, is not safe: it yields a fractured part. On the contrary, the case of global annealing $\mathrm{f}$ ) is satisfactory, as demonstrated by the results of experiments and predicted by simulations. 
If focusing on the cases with local annealing, the corresponding FLD plots are given in Figure 10. Case c) is not satisfactory, as it induces fracture of the tube. Cases b) and e), where conical regions have been treated, are safe and clearly show a discontinuity in the distribution of elemental principal strain values on the left quadrant of the FLD plot. This "hole" in the diagram is bigger for case e), due to a larger conical treated region. Case d) is safe too, but there is a clear group of elements, represented by yellow dots, which is undergoing a potentially dangerous plane strain deformation (minor strain nearly null) with a non-negligible major strain. For this reason, solution d) cannot be considered sufficiently reliable. The choice of the best solution between cases $b$ ) and e) relies on the type of heat source that can be used for annealing. If a beam technology is used, as a laser beam, case b) is preferable since case e) requires a prolonged scanning time and a complicated setup. If an induction heater is used, a simple solution can be a ring-shaped heater around the tube, and option e) seems preferable.

(b)

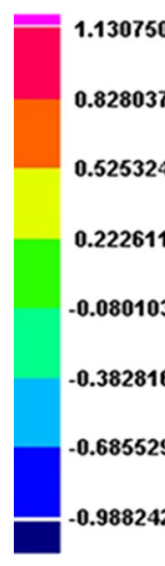

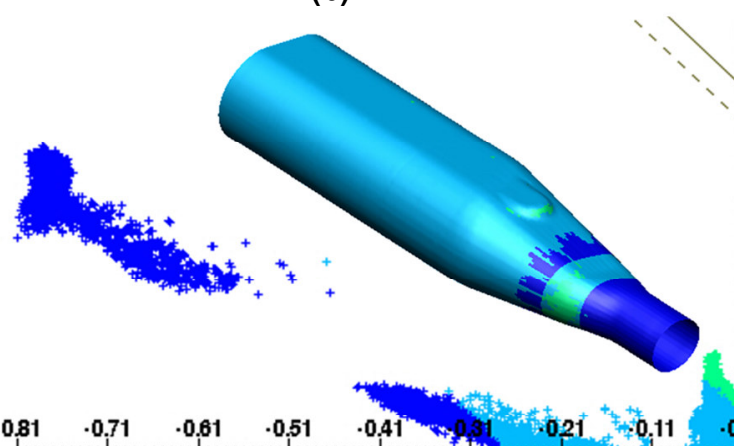

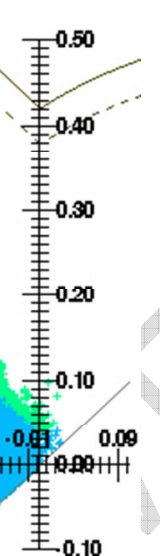

(d)

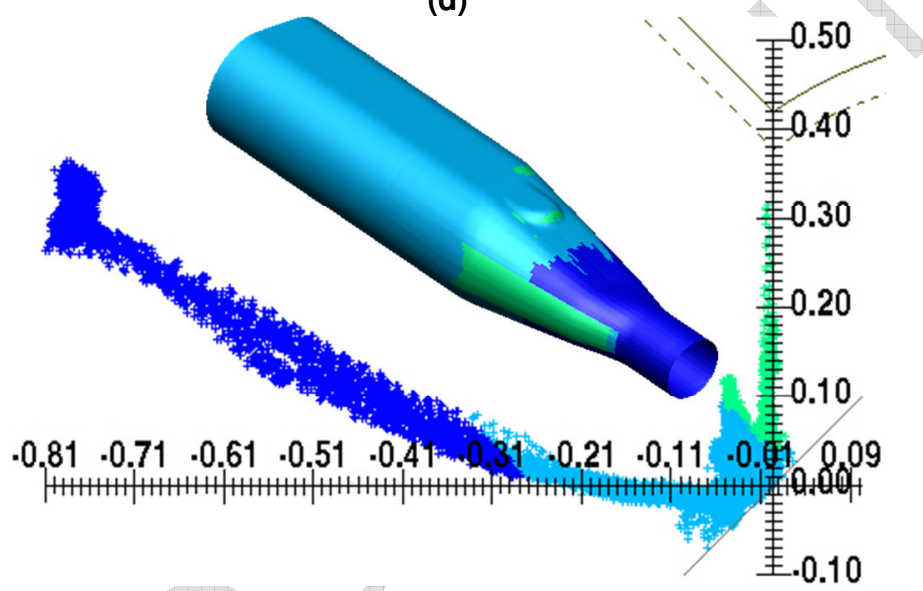

(c)

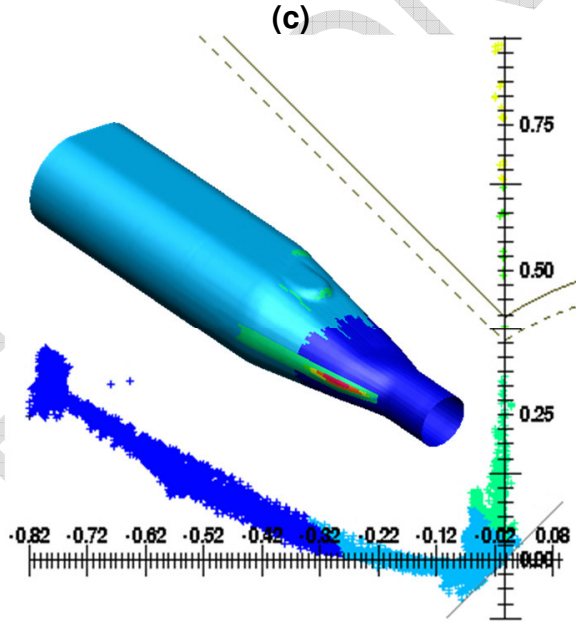

(e)

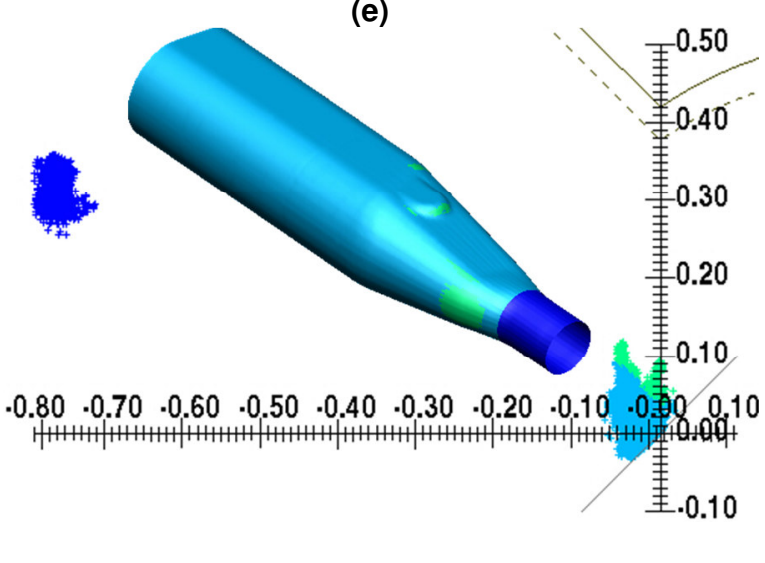

Figure 10: risk of fracture for four investigated options, red dots indicate elements with high risk of fracture.

\subsection{Shape calibration}

From the previous subsection cases b) and e) emerge as the only two viable options to the traditional solution of global annealing in a furnace, which is case f). Considering the capability of calibrating the shape of the semi-dome, the cross-sectional profile of cases b), and e) are shown in Figure 11, in which the external discretized contour is represented as $x$ and $y \_o u t$ coordinates. The calculated values of the calibrated minimum radius $R_{\min }$, are given in Table 4 . Cases e) and f), respectively annealing in a conical region and global annealing yield similar results, significantly better than case b), which is less calibrated, with about $1 \mathrm{~mm}$ greater $R_{\min }$ value. This is probably due to the material around the small annealed patch which is hardened by the full process sequence and determines a more rigid behavior of the whole tube in calibration. 


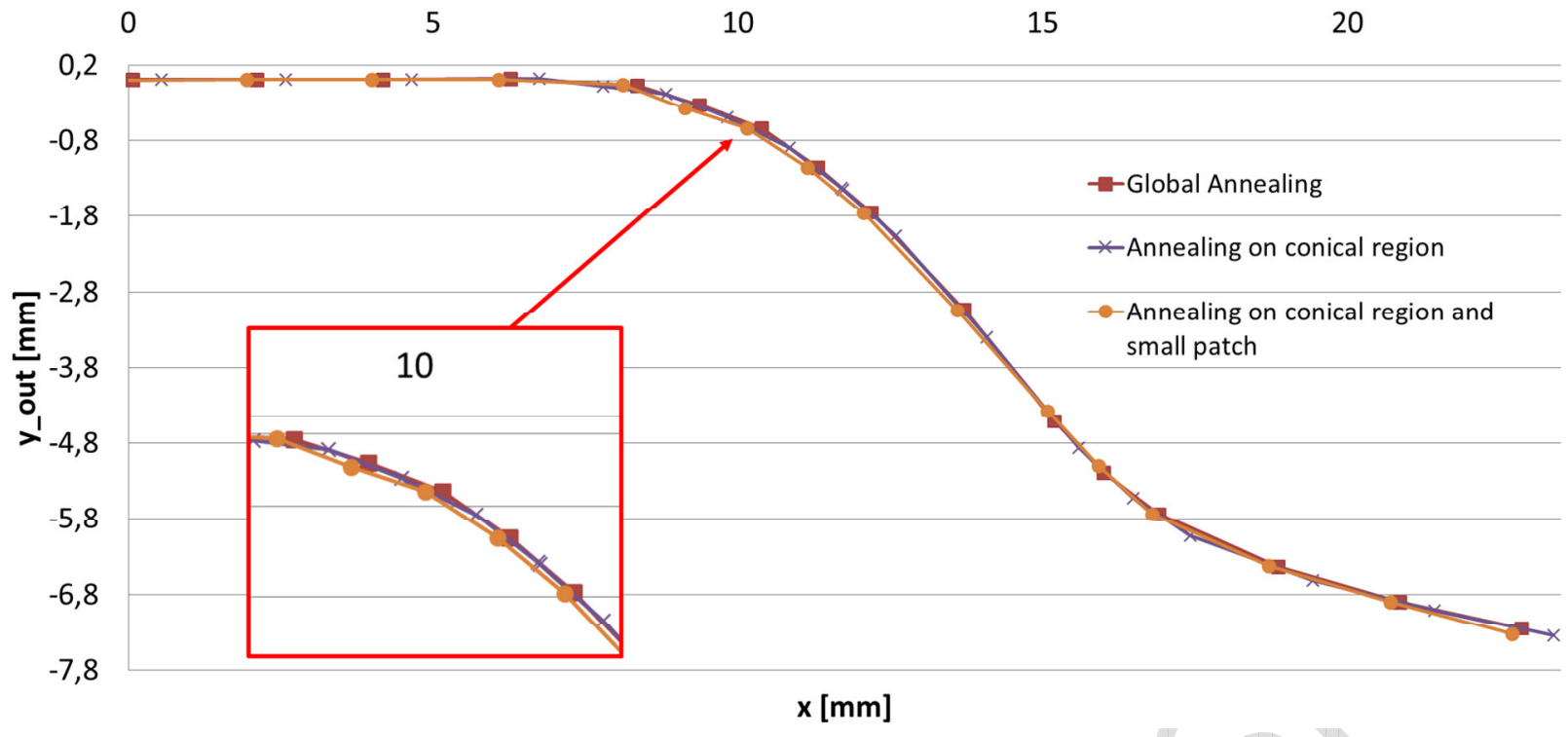

Figure 11: shape comparison at the semi-dome location.

\begin{tabular}{|l|c|c|}
\hline & $\begin{array}{c}\text { FEM calculated } R_{\min } \\
{[\mathrm{mm}]}\end{array}$ & $\begin{array}{c}\text { Experimental } R_{\min } \\
{[\mathrm{mm}]}\end{array}$ \\
\hline Case b) annealing on conical region and small patch & 8.4 & n.a. \\
\hline Case e) annealing on conical region & 7.7 & n.a. \\
\hline Case f) global annealing & 7.8 & 5.6 \\
\hline
\end{tabular}

Table 4: comparison of experimental and simulated $\mathbf{R}_{\min }$

\subsection{Options for local annealing methods}

The previous subsections clearly demonstrated that two strategies can be effectively used for local annealing: treating the tube on a relatively large annular region (case e), treating the tube on relatively small but carefully designed patches (case b).

In case a precise and localized heating technology is required, a laser beam could be used for scanning the material and locally annealing it. In order to keep the total scanning time (and cost) under prescribed limit and in order for the proposed innovation to be competitive to the traditional furnace annealing, a solution where small portion of the tube must be chosen, if possible. In the proposed example, case b), where the heat treatment is performed strictly on the two local regions where the risks of fracture and insufficient calibration are present, represents the suitable solution. Experiments of local laser annealing of the $\mathrm{Al} 6060$ tubes have been performed at the SITEC research center at Politecnico di Milano, with a $1 \mathrm{~kW}$ fiber laser. The laser treating tests have shown that a complete annealing can be obtained on the Al 6060 alloy, with full recovery of the original material formability.

In the proposed example, if a very good die filling is desired, in addition to the improvement of ductility, the strategy of locally treating a relatively large portion of the tube (case e) must be preferred. Induction heating or infrared heating are less precise and localized methods and they are suited for treating larger portions. Induction heaters for aluminum tubes are commercially available [28] [27] and infrared heaters have been tested too for alloys of the 6XXX series, although complete annealing is difficult to achieve in this case [29].

\section{Conclusions}

In this paper, the possibility of improving the tube hydroforming process chain by substituting the conventionally adopted global intermediated annealing, which is performed in furnaces, with an innovative local annealing strategy has been investigated. THF experiments and simulations have been carried out on tubes with and without global annealing, and the accuracy and reliability of FEM simulations in predicting the risk of failure by fracture and the die filling have been assessed. Then, FEM simulations have been used for 
comparing the outcome of several different local annealing strategies. The results clearly show that local treatment, for the studied case, is a feasible option.

If a localized beam technology (e.g. a laser beam) can be used for the heat treatment, a solution which targets only relatively small areas of the tube can be effectively adopted. This strategy improves formability of the material where needed, but may not yield the desired degree of calibration against the die.

Alternatively, if a more diffused heating solution is available (e.g. an induction heating ring), larger patches of the tube can be effectively adopted. This improves formability and facilitates calibration.

\section{Acknowledgments}

The authors wish to thank and acknowledge the co-operation of Maspero of Monza (Italy), especially Mr. Daniele Carra, for the hydroforming experiments and the research center on laser processing SITEC at Politecnico di Milano for the laser annealing trials. Stefano Zarini and Ehsan Mostaed of Politecnico are thankfully acknowledged for their help respectively in the laser tests and the tube material characterization.

This research has been conducted within the project "Origami", which is partly financed by the Regione Lombardia and scientifically coordinated by Prof. M. Vedani of Politecnico di Milano.

\section{References}

[1] Schlemmer KL, Osman FH. Differential heating forming of solid and bi-metallic hollow parts. Journal of Materials Processing Technology 2005;162-163:564-9.

[2] Matzenmiller A, Bröcker C. Thermo-mechanically coupled FE analysis and sensitivity study of simultaneous hot/cold forging process with local inductive heating and cooling. International Journal of Material Forming 2011;5:275-300.

[3] Yoshihara S, MacDonald BJ, Nishimura H, Yamamoto H, Manabe K. Optimisation of magnesium alloy stamping with local heating and cooling using the finite element method. Journal of Materials Processing Technology 2004;153-154:319-22.

[4] Li J, Zhou Y, Hu SJ, Izquierdo LE, Krajewski PE, Lee TM. Optimization of localized annealing for preform anneal forming of aluminum alloys. Transactions of the North American Manufacturing Research Institution of SME, vol. 38, Department of Mechanical Engineering, University of Michigan, Ann Arbor, MI, United States: 2010, p. 411-8.

[5] Figala G, Buchmayr B. Improvement of buckling resistance of thin sheets by local laser treatment. International Journal of Material Forming 2010;3:829-32.

[6] Tönshoff HK, Bunte J, Meier O, Engelbrecht L. Deformation behaviour of sheet metals in laserassisted hydroforming processes. Advanced Materials Research 2005;6-8:361-8.

[7] Fan G, Gao L, Hussain G, Wu Z. Electric hot incremental forming: A novel technique. International Journal of Machine Tools and Manufacture 2008;48:1688-92.

[8] Strano M, Del Prete A, Primo T, Mentella A. Localized warming of sheet metal parts for the reduction of springback. Numisheet 2008, Interlaken: 2008.

[9] Mäntyjärvi K, Keskitalo M, Karjalainen JA, Leiviskä A, Heikkala J, Mäkikangas J. Laser-Assisted Bending. Key Engineering Materials 2007;344:235-41.

[10] Walczyk DF, Vittal S. Bending of Titanium Sheet Using Laser Forming, Journal of Manufacturing Processes 2000; 2: 258-69.

[11] Hu Z. Elasto-plastic solutions for spring-back angle of pipe bending using local induction heating. Journal of Materials Processing Technology 2000;102:103-8.

[12] Geiger M, Merklein M, Pitz M. Laser and forming technology-an idea and the way of implementation. Journal of Materials Processing Technology 2004;151:3-11.

[13] Okman O, Ozmen M, Huwiler H, Tekkaya A. Free forming of locally heated specimens. International Journal of Machine Tools and Manufacture 2007;47:1197-205.

[14] Duflou JR, Callebaut B, Verbert J, De Baerdemaeker H. Laser Assisted Incremental Forming: Formability and Accuracy Improvement. Annals of the CIRP 2007;56:273-6.

[15] Lee H-W, Bae J-H, Kim M-S, Kim C. Optimum design of pipe bending based on high-frequency induction heating using dynamic reverse moment. International Journal of Precision Engineering and Manufacturing 2011;12:1051-8. 
[16] Sun X, Smith LM. External heating closed-volume thermally activated tube forming: A fundamentally new approach for hydroforming thick-walled tubes. Journal of Manufacturing Processes 2010;12:63-6.

[17] Palumbo G, Sorgente D, Tricarico L. The design of a formability test in warm conditions for an AZ31 magnesium alloy avoiding friction and strain rate effects. International Journal of Machine Tools and Manufacture 2008;48:1535-45.

[18] Romero P, Otero N, Cabrera JM, Masagué D. Laser assisted conical spin forming of dual phase automotive steel. Experimental demonstration of work hardening reduction and forming limit extension. Physics Procedia 2010;5:215-25.

[19] Haferkamp H, Bunte J, Engelbrecht L. Material characterisation for laser-assisted sheet metal hydroforming. MPMD Sixth Global Innovations Proceedings - Trends in Materials and Manufacturing Technologies for Transportation Industries and Powder Metall. Research and Development in the Transportation Industry, San Francisco: 2005, p. 83-8.

[20] Järvenpää $A$, Jaskari $M$, Karjalainen LP, Hietala M. Enhancing mechanical properties and formability of AISI 301LN stainless steel sheet by local laser heat treatment. Key Engineering Materials 2013;554-557:885-92.

[21] Capello E, Previtali B. Enhancing dual phase steel formability by diode laser heat treatment. Journal of Laser Applications 2009;21:1.

[22] Neugebauer PR, Schieck F, Werner M. Tube press hardening for light weight design. Proceedings of the ASME 2011 International Manufacturing Science and Engineering Conference, Corvallis, USA: 2011.

[23] Sulzberger, Merklein M, Staufner, Wortberg. Methodology to produce locally heat treated 5182 Aluminium alloy Sheet Metal Parts. Key Engineering Materials 2012;504-506:113-8.

[24] Staud D, Merklein M. Inverse Approach to the Forming Simulation of Tailored Heat Treated. International Journal of Material Forming 2008;Suppl 1:37-40.

[25] Strano M, Jirathearanat S, Shr S-G, Altan T. Virtual process development in tube hydroforming. Journal of Materials Processing Technology 2004;146:130-6.

[26] Strano M. Design and modeling of parts, process and tooling in tube hydroforming. In: Koç M, editor. Hydroforming for advanced manufacturing, Cambridge, UK: Woodhead Publishing Limited; 2008.

[27] Kalpakjian S. Dimensional Changes in Tube Swaging. Journal of Engineering for Industry 1966;88:147.

[28] Dawei Induction Heating Machine Co., Ltd http://www.dw-inductionheating.com/annealing-aluminumtubing.html.

[29] Rivard, J.D.K., Blue, C.A., Ott, R.D., Sabau, A., Santella, M., Pan, T.-Y., Joaquin, A., 2004. Advanced Manufacturing Technologies Utilising High Density Infrared Radiant Heating. Surface Engineering 20, 220-228. 